\title{
AUUSTE DE MODELOS DE TAPER E SORTIMENTO DE TORAS DE MOGNO AFRICANO EM SISTEMAS AGROFLORESTAIS EM BELTERRA, PARÁ
}

\author{
Lucas Sérgio de Sousa Lopes; ; Rafael Rode'; Daniela Pauletto ${ }^{3}$; Diego Damázio Baloneque ; Arystides \\ Resende da Silva ${ }^{5}$; Ketlen Naiane Freitas dos Santos ${ }^{6}$. \\ ${ }^{1}$ Engenheiro Florestal, Santarém, Pará, Brasil, lucaasergio@gmail.com \\ ${ }^{2}$ Universidade Federal do Oeste do Pará, Santarém, Pará, Brasil, rafaelrode@gmail.com \\ ${ }^{3}$ Universidade Federal do Oeste do Pará, Santarém, Pará, Brasil, danielapauletto@hotmail.com \\ ${ }^{4}$ Baloneque Ambiental, Placas, Pará, Brasil, balonequedd@gmail.com \\ ${ }^{5}$ Empresa Brasileira de Pesquisa Agropecuária, Belém, Pará, Brasil, arystides.silva@embrapa.br \\ ${ }^{6}$ Engenheira florestal, Santarém, Pará, Brasil, ketlennayane@gmail.com
}

RESUMO: A utilização de modelos de taper possibilita a estimativa do número de toras e seus respectivos volumes, de acordo com a destinação da madeira. Diante disso, o objetivo do presente trabalho foi ajustar modelos de taper e estimar a partir destes, o número de toras de mogno africano (Khaya ivorensis A. Chev.) geradas em sistemas agroflorestais em Belterra, Pará. A coleta de dados para elaboração deste estudo se deu em dois sistemas agroflorestais no município de Belterra, onde cubouse 35 árvores em cada um dos locais. Foram ajustados 3 modelos de taper para os dois sistemas, o melhor modelo foi escolhido através de estatísticas de qualidade do ajuste e tomado para utilização na estimativa sortimento dos multiprodutos das árvores. Para o sortimento, estabeleceu-se três classes de dimensões das toras de acordo com a utilização, sendo: C1 = Tora para energia com diâmetro na ponta fina maior ou igual a $15 \mathrm{~cm}$ e 2 metros de comprimento; $\mathrm{C2}$ = Tora para serraria com diâmetro na ponta fina maior ou igual a $20 \mathrm{~cm}$ e 2 metros de comprimento; $\mathrm{C} 3$ = Tora para serraria com diâmetro na ponta fina maior ou igual a $25 \mathrm{~cm}$ e 2 metros de comprimento. O modelo de Kozak foi o que apresentou o melhor desempenho para estimativa do afilamento do fuste, com a raiz quadrada do erro médio (RQEM\%) inferior nos dois locais. Quanto ao sortimento, os sistemas estudados podem geram de 47 a 163 toras de madeira, de acordo com o cenário e o local, sendo a utilização para fins de energia a que confere maior volume efetivamente utilizado.

PALAVRAS-CHAVE: Agrossilvicultura, Manejo florestal, Volumetria.

\section{ADJUSTMENT OF TAPER MODELS AND ASSORTMENT OF LOGS OF AFRICAN MAHOGANY IN AGROFORESTRY SYSTEMS IN BELTERRA, PARÁ}

ABSTRACT: The use of taper models makes it possible to estimate the number of logs and their respective volumes according to the destination of wood. Therefore, the objective of the present study was to adjust taper models and to estimate the number 
of African mahogany logs (Khaya ivorensis A. Chev.) Generated in agroforestry systems in Belterra, Pará. Data collection for this study was done in two agroforestry systems in the municipality of Belterra, where 35 trees were covered in each one of the sites. Three models of taper were adjusted for both systems, the best model was chosen through adjustment quality statistics and taken for use in estimating the assortment of the multiproducts of the trees. For the assortment, three classes of log dimensions were established according to the use, being: $\mathrm{C} 1$ = Tora for energy with a diameter at the thin point greater than or equal to $15 \mathrm{~cm}$ and 2 meters in length; $\mathrm{C} 2$ = Tora for sawmill with diameter at the thin point greater or equal to $20 \mathrm{~cm}$ and 2 meters in length; C3 = Saw blade with a fine-tip diameter of $25 \mathrm{~cm}$ or more and 2 meters in length. The Kozak model was the one that presented the best performance for the estimation of the bole taper, with the square root of the mean error (SRME\%) lower at both sites. As for the assortment, the systems studied can generate from 47 to 163 wood logs, according to the scenario and the place, being the use for energy purposes that gives the largest volume actually used.

KEYWORDS: Agroforestry, Forest management, Volumetry.

\section{AJUSTE DE MODELOS DE TAPER Y SURTIDO DE TRONCOS DE CAOBA AFRICANA EN SISTEMAS AGROFORESTALES EN BELTERRA, PARÁ}

RESUMEN: La utilización de modelos de taper posibilita la estimación del número de troncos y sus respectivos volúmenes, de acuerdo con el destino de la madera. Frente a ello, el objetivo del presente trabajo fue ajustar modelos de taper y estimar a partir de éstos, el número de troncos de caoba africana (Khaya ivorensis A. Chev.) Generados en sistemas agroforestales en Belterra, Pará. La recolección de datos para la elaboración de este estudio se dio en dos sistemas agroforestales en el municipio de Belterra, donde se cubrieron 35 árboles en cada uno de los locales. Se ajustaron 3 modelos de taper para los dos sistemas, el mejor modelo fue elegido a través de estadísticas de calidad del ajuste y tomado para su uso en la estimación surtida de los multiproductos de los árboles. Para el surtido, se establecieron tres clases de dimensiones de las tuercas de acuerdo con la utilización, siendo: $\mathrm{C} 1$ = Tora para energía con diámetro en la punta fina mayor o igual a $15 \mathrm{~cm}$ y 2 metros de longitud; C2 = Tora para aserrar con diámetro en la punta fina mayor o igual a $20 \mathrm{~cm}$ y 2 metros de longitud; C3 = Tora para aserrar con diámetro en la punta fina mayor o igual a 25 cm y 2 metros de longitud. El modelo de Kozak fue el que presentó el mejor desempeño para la estimación del afilado del fuste, con la raíz cuadrada del error medio (RCEM\%) inferior en los dos locales. En cuanto al surtido, los sistemas estudiados pueden generar de 47 a 163 troncos de madera, de acuerdo con el 
escenario y el local, siendo la utilización para fines de energía a la que confiere mayor volumen efectivamente utilizado.

PALABRAS CLAVE: Agrosilvicultura, Manejo forestal, Volumetría.

\section{INTRODUÇÃO}

O mogno africano (Khaya ivorensis

A. Chev.) é uma essência florestal nativa das regiões de florestas tropicais úmidas da África Ocidental, tal espécie, tem sido cada vez mais introduzida no Brasil, principalmente como componente arbóreo de sistemas agroflorestais (SAFs), graças ao seu rápido ritmo de crescimento e ao elevado valor econômico de sua madeira, altamente apreciada no mercado interno e externo (PINHEIRO et al., 2011; RIBEIRO et al., 2017).

Considerando a importância da espécie e tendo em vista a necessidade do suprimento de madeiras para abastecer o mercado, quantificar $\mathrm{O}$ estoque de toras e conhecer suas distintas possibilidades de uso torna-se fundamental para o manejo adequado dos sistemas agroflorestais, pois fornece informaç̃̃es quantitativas e qualitativas, auxiliando a avaliação técnica e econômica dos plantios (SCHRÖDER et al., 2013; CORDEIRO et al., 2015).

Uma ferramenta eficaz para tal, é o estudo da forma dos fustes das árvores, também conhecido como afilamento, comumente realizado através das modelos de taper, cuja funcionalidade baseia-se na representação matemática da forma do fuste das árvores, possibilitando a estimativa do diâmetro para qualquer altura ao longo do fuste ou vice-versa, bem como do volume das árvores (CAMPOS; LEITE, 2017).

As equações geradas através do ajuste dos modelos de taper possibilitam a estimativa do número de toras e seus respectivos volumes, a partir das dimensões especificadas de acordo com a destinação da madeira, permitindo assim simular cenários de custo e renda os quais podem auxiliar na definição da rotação da floresta e na tomada de decisão (COSTA et al., 2016). 
Diante do exposto, o objetivo do presente trabalho foi ajustar modelos de taper e estimar a partir destes, o número de toras de Khaya ivorensis $\mathrm{A}$. Chev. geradas em sistemas agroflorestais em Belterra, Pará.

\section{MATERIAL E MÉTODOS}

A coleta de dados para elaboração deste estudo se deu em dois sistemas agroflorestais no município de Belterra, Pará (Figura 1) situado no Oeste do estado. Segundo classificação de Köppen o clima da região é do tipo Am, com precipitação média de aproximadamente $1.743 \mathrm{~mm}$ e temperatura anual média de $25^{\circ} \mathrm{C}$. $\mathrm{O}$ solo é predominantemente formado por Latossolo Amarelo de textura argilosa (ESPÍRITO-SANTO et al., 2005).

Figura 1. Localização das áreas de estudo.

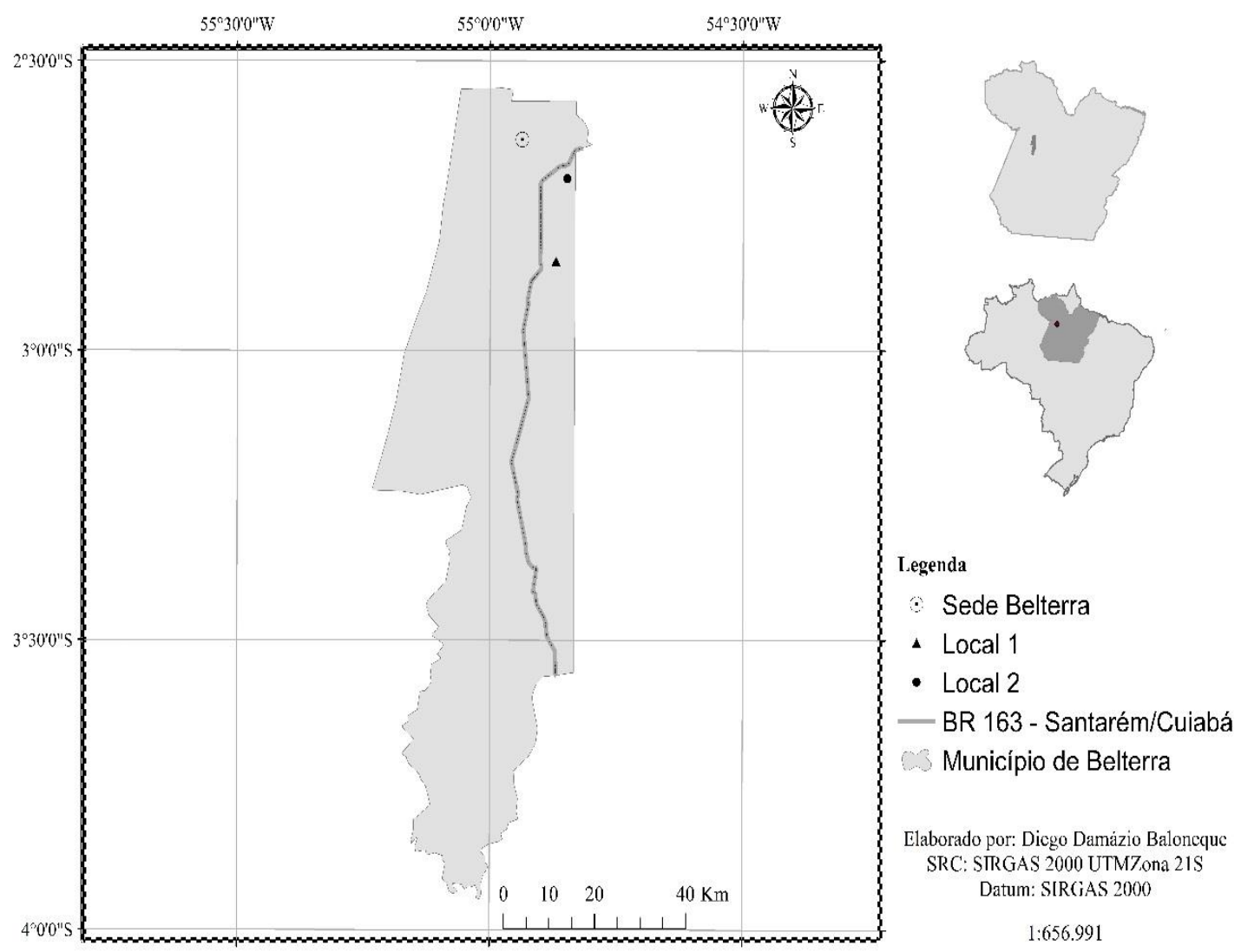


No local 1 está inserido um sistema de integração lavoura-pecuária-floresta (iLPF), com 7 anos de idade e espaçamento de $7 \times 5 \mathrm{~m}$ entre as árvores de mogno africano, onde houve cultivo de espécies agrícolas e pastejo de gado bovino anteriormente. As árvores estão distribuídas em 8 linhas de plantio. No local 2 está implantado um sistema silvipastoril, composto por gado bovino e pastagens, mantido há 11 anos, onde as árvores de mogno africano estão dispostas em espaçamento de 10 × 10 m e agrupadas em um renque único, composto por duas filas de plantio.

Foram cubadas 35 árvores em pé de cada local, utilizando o dendrômetro Criterion RD 1000. Aferiu-se o diâmetro a 0,1, 0,5, 1 e 1,30 m (Diâmetro a altura de
1,3 m - DAP) de altura acima do solo em todas as árvores. A partir da altura de 2,0 $m$, prosseguiu-se a cubagem com o dendrômetro de metro em metro até a bifurcação, onde considerou-se como altura comercial $(\mathrm{Hc})$, conforme ilustrado na Figura 2. A altura total das árvores $(\mathrm{Ht})$ foi estimada com auxílio de hipsômetro Vertex IV e o volume do fuste, bem como das seções, foram obtidos através do método de Smalian

Foram selecionados para ajuste 3 modelos de taper amplamente difundidos na literatura (Tabela 1). Os ajustes foram realizados utilizando o algoritmo Gauss-Newton, mediante o método de estimativa dos mínimos quadrados não lineares.

Figura 2. Ilustração do procedimento de cubagem de árvore-amostra.

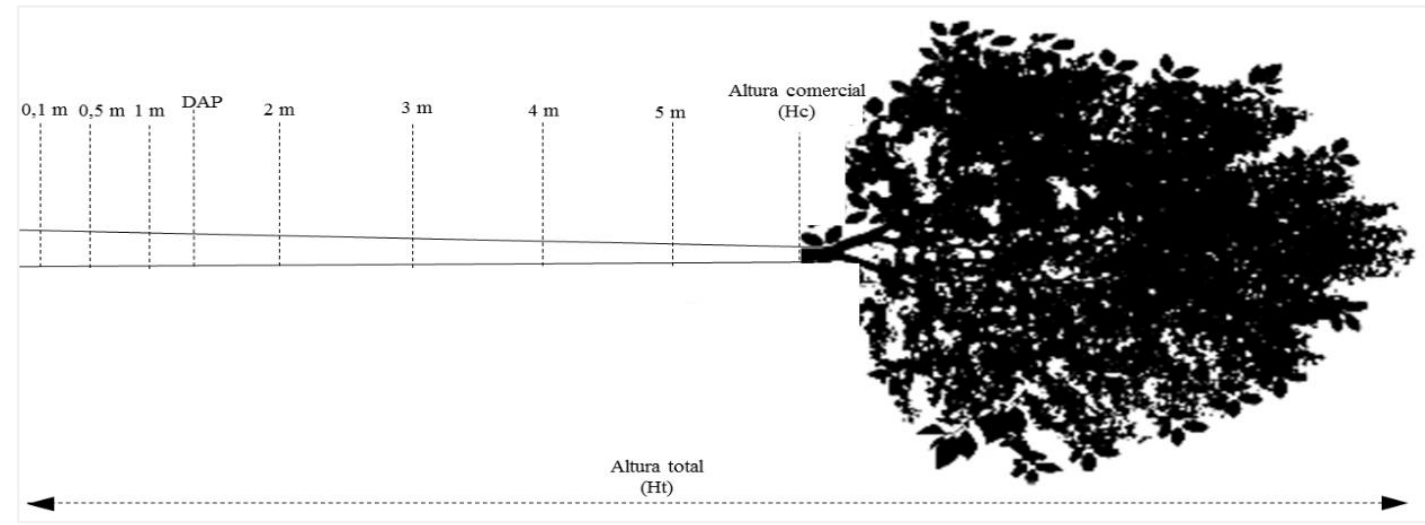


Tabela 1. Modelos de taper ajustados para Mogno Africano (Khaya ivorensis A. Chev.) em dois sistemas agroflorestais em Belterra, Pará.

\begin{tabular}{cc}
\hline Autor & Modelo \\
\hline Kozak & $\left(\frac{d_{i}}{D A P}\right)^{2}=\beta_{0}+\beta_{1}\left(\frac{h_{i}}{H T}\right)+\beta_{2}\left(\frac{h_{i}}{H T}\right)^{2}+\varepsilon$ \\
Demaerschalk & $\left(\frac{d_{i}}{D A P}\right)^{2}=10^{2 \beta_{0}} D A P^{\left(2 \beta_{1}-2\right)} H T^{2 \beta_{2}}\left(H T-h_{i}\right)^{2 \beta_{3}}+\varepsilon$ \\
Ormerod & $\left(\frac{d_{i}}{D A P}\right)^{2}=D A P\left(\frac{H T-h_{i}}{H T-1,3}\right)^{2 \beta_{1}}+\varepsilon$
\end{tabular}

$\overline{\mathrm{DAP}}=$ diâmetro à $1,3 \mathrm{~m}$ do solo em $\mathrm{cm} ; d_{i}=$ diâmetro na altura $h_{i}$ em $\mathrm{cm} ; \mathrm{HT}=$ altura total em $\mathrm{m} ; h_{i}$ = altura da seção em m; $\beta_{i}=$ parâmetros do modelo; $\varepsilon=$ erro aleatório.

A seleção do modelo de taper a ser utilizado para estimar os multiprodutos do sortimento de toras de Mogno africano foi conduzida através dos parâmetros de qualidade de ajuste mencionados por Campos e Leite (2017): Coeficiente de correlação entre os valores estimados e observados de afilamento $\left(r_{y \hat{y}}\right)$; raiz quadrada do erro médio em porcentagem (RQEM\%) e coeficiente de determinação ajustado ( $r^{2}$ aj.), equações $1 ; 2$ e 3, respectivamente.

$$
\begin{gathered}
r_{y \hat{y}}=\frac{\operatorname{cov}(Y, \hat{Y})}{\sqrt{s^{2}(Y) s^{2}(\bar{Y})}} \\
R Q E M \%=\frac{100}{\bar{Y}} \sqrt{\frac{\sum(d i f f)^{2}}{n}} \\
r^{2} a j .=1-\left[\left(1-r^{2}\right)\left(\frac{n-1}{n-p-1}\right)\right.
\end{gathered}
$$

Em que: cov = covariância; $\widehat{Y}_{i}=$ valor estimado da variável para a $i$-ésima árvore; $Y_{i}=$ valor observado da variável na i-ésima árvore; $\mathrm{n}$ = número total de observações; $\mathrm{p}=$ número de variáveis independentes; $\bar{Y}=$ média dos valores observados da variável $Y_{;} \mathrm{s}^{2}=$ desvio padrão; diff ${ }_{i}=\left(Y_{i}\right.$ estimado - Yi observado). 
Para quantificação do número de toras geradas nos sistemas agroflorestais pesquisados, adotou-se 3 classes de sortimento de madeira:

C1 = Tora para fins energéticos com diâmetro na ponta fina maior ou igual a $15 \mathrm{~cm}$ e 2 metros de comprimento; C2 = Tora para serraria com diâmetro na ponta fina maior ou igual a $20 \mathrm{~cm} \mathrm{e}$ 2 metros de comprimento; $\mathrm{C} 3$ = Tora para serraria com diâmetro na ponta fina maior ou igual a $25 \mathrm{~cm}$ e 2 metros de comprimento.
Cada classe de sortimento foi modelada para os dois sistemas, perfazendo 6 cenários. A estimativa volumétrica das toras foi realizada com a integral da equação gerada pelo melhor modelo de taper.

\section{RESULTADOS E DISCUSSÃO}

Os resultados dos ajustes e coeficientes das equações de taper estão contidos na Tabela 2.

Tabela 2. Estimativas dos parâmetros e estatísticas de ajuste dos modelos de taper ajustados para Mogno africano (Khaya ivorensis A. Chev.) em dois sistemas agroflorestais em Belterra, Pará.

\begin{tabular}{ccccccccc}
\hline Local & Modelo & $\beta_{0}$ & $\beta_{1}$ & $\beta_{2}$ & $\beta_{3}$ & $r_{y \hat{y}}$ & RQEM\% & $r^{2}$ aj. \\
\hline \multirow{4}{*}{ Local 1 } & Demaerschalk & 0,2356 & 0,8817 & $-0,1489$ & 0,1376 & 0,82 & 2,43 & 0,71 \\
& Kozak & 1,3719 & $-2,0640$ & 1,6335 & - & 0,88 & 2,02 & 0,83 \\
& Ormerod & 1,2000 & 0,1711 & - & - & 0,76 & 2,82 & 0,55 \\
\multirow{4}{*}{ Local 2 } & Demaerschalk & 0,1055 & 0,9982 & $-0,2175$ & 0,1877 & 0,95 & 3,00 & 0,76 \\
& Kozak & 1,2345 & $-2,1651$ & 2,1604 & - & 0,96 & 2,61 & 0,83 \\
& Ormerod & - & 0,3071 & - & - & 0,94 & 3,15 & 0,91 \\
\hline
\end{tabular}

$r_{y \hat{y}}=$ Coeficiente de correlação entre os valores estimados e observados de afilamento; RQEM\% = raiz

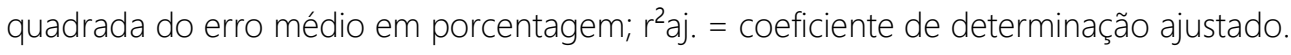

Para o local 1, o modelo de Kozak foi o que apresentou as melhores estatísticas de qualidade de ajuste, demonstrando superioridade em todos os critérios utilizados para inferir sobre o ajuste. No entanto, para o local 2 o modelo de Ormerod apresentou um $r^{2}$ aj. superior aos demais, porém nos outros parâmetros avaliados, o modelo de 
Kozak novamente obteve destaque, apresentando um RQEM\% baixo e um alto coeficiente de correlação. Desse modo, tomou-se o modelo de Kozak para as estimativas de sortimento.

Ao utilizarem modelos de taper tradicionalmente difundidos, Campos et al. (2017) atestaram que Demaerschalk e Ormerod demonstraram desempenho pouco satisfatório, com valores de estatísticas de ajustes inferiores aos demais modelos. Quanto aos valores de $r^{2}$ aj, Campos et al., (2014) comparando 5 modelos de taper, obteve os menores valores de coeficiente de determinação para Demaerschalk e Ormerod.

Costa et al. (2016) encontraram as melhores estimativas do afilamento de Araucaria angustifolia utilizando o modelo de Kozak, bem como, o utilizaram para estimar o sortimento de toras desta espécie. Leite et al. (2014) ao compararem as estimativas geradas pelo modelo de Kozak com redes neurais artificiais, atestaram boa precisão desse modelo, com pequena tendência a superestimava. Segundo Campos e Leite (2017) o modelo de Kozak é o mais difundido no meio florestal devido a sua facilidade de ajustes e por gerar estimativas com boa precisão. A escolha de modelos mais precisos para estimativa da forma do fuste é fundamental, uma vez que o ganho em precisão nas estimativas de árvores individuais reflete diretamente em ganhos significativos quando levados para nível de povoamento (CORDEIRO et al., 2015).

A Figura 3 representa o perfil do fuste das árvores de Mogno africano dos dois sistemas agroflorestais, com os valores observados e estimados pelas equações ajustadas para as áreas. Nota-se que o modelo apresentou uma melhor distribuição de valores estimados para o local 2. Ao passo que, para o local 1 o modelo proporcionou certa tendenciosidade a subestimação do afilamento nas seções intermediárias do fuste e sobrestimação nas seções inferiores. Em ambos os locais, os fustes apresentaram pouca conicidade ao longo de quase toda sua extensão e formato neiloide na base do fuste, corroborando com Pinheiro et al. (2011), que descreve o fuste das árvores de mogno africano como predominantemente retilíneo.

A Tabela 3 contém os cenários simulados a partir das classes de sortimento propostas para os dois locais pesquisados. A 
quantidade de toras geradas variou entre 47

e 163 e o volume médio entre 0,0942 $\mathrm{m}^{3}$ a

$0,2127 \mathrm{~m}^{3}$.

Observa-se que o maior aproveitamento de volume se dá na C1, devido as especificações diamétricas estabelecidas serem de mais fácil atendimento, gerando assim maior quantidade de toras e maior volumetria comercial.

Figura 3. Dispersão dos dados de di/DAP em função de hi/HT observados e estimados pelo modelo de Kozak para as árvores de Mogno africano (Khaya ivorensis A. Chev.) em dois sistemas agroflorestais em Belterra, Pará.
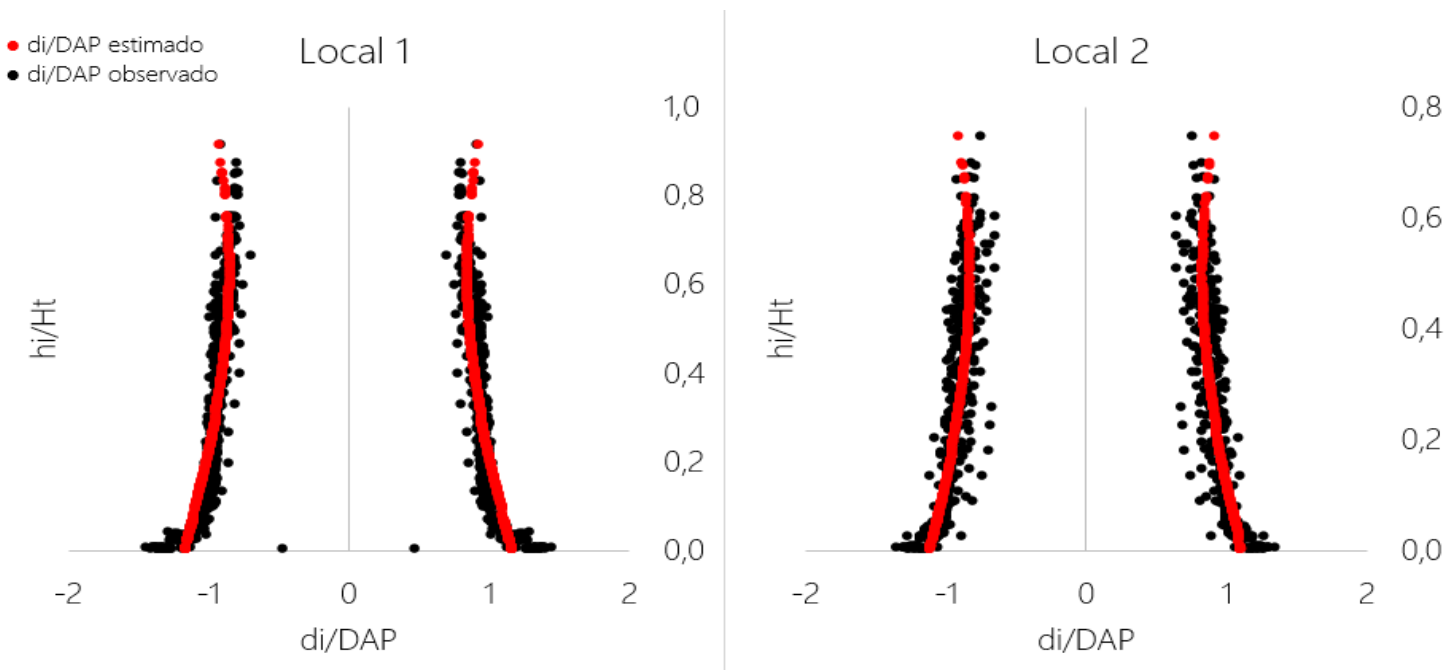

Tabela 3. Sortimento de madeira do fuste de Mogno africano (Khaya ivorensis A. (hev.) em dois sistemas agroflorestais em Belterra, Pará.

\begin{tabular}{cccccc}
\hline Classe & Local & N de toras & $V_{\text {médio }}\left(\mathrm{m}^{3}\right)$ & $V_{\text {comercial }}\left(\mathrm{m}^{3}\right)$ & $V_{\text {residual }}\left(\mathrm{m}^{3}\right)$ \\
\hline \multirow{2}{*}{ C1 } & Local 1 & 163 & 0,0942 & 15,3508 & 1,0826 \\
& Local 2 & 136 & 0,1832 & 24,9184 & 2,7538 \\
\multirow{2}{*}{ C2 } & Local 1 & 135 & 0,1005 & 13,5646 & 1,2232 \\
& Local 2 & 126 & 0,1932 & 24,3461 & 2,8622 \\
\multirow{2}{*}{ C3 } & Local 1 & 47 & 0,1722 & 8,0940 & 1,2328 \\
& Local 2 & 107 & 0,2127 & 22,7627 & 2,7193 \\
\hline
\end{tabular}

$\mathrm{N}=$ número; $\mathrm{V}_{\text {médio }}=$ Volume com casca médio por tora de madeira; $\mathrm{V}_{\text {comercial }}=$ Volumetria total dentro das especificações comerciais da classe de sortimento e $V_{\text {residual }}=$ Volumetria total fora das especificações comerciais da classe de sortimento. 
Considerou-se como volume residual as toras que não apresentam dimensões especificadas nas classes de sortimento, por se tratar em geral de pontas de fuste com diâmetros inferiores aos explorados. No entanto, essa volumetria pode também ser destinada a energia, para gerar ganhos econômicos aos proprietários da área, conforme explicitado por Chichorro et al. (2003).

Em todos os cenários, o local 2 forneceu maior número de toras e maior volumetria comercial aproveitável, fato este que pode ser atribuído a idade das árvores presentes nesse sistema, uma vez que árvores mais velhas fornecem uma quantidade maior de toras, devido suas dimensões, conforme atestado por Figueiredo Filho et al. (2015) para Araucaria angustifolia.

\section{CONCLUSÃO}

Os modelos de taper ajustados para as árvores de mogno africano em sistemas agroflorestais apresentaram qualidade de ajuste, com destaque para o modelo de Kozak.

As árvores de mogno africano sistemas agroflorestais podem gerar diferentes números de toras de madeira, de acordo com o uso requerido.

A utilização para fins energéticos gera o maior número de toras, ao passo que, a utilização para serraria a partir de $25 \mathrm{~cm}$ de ponta fina de diâmetro, a maior volumetria média por tora das árvores de mogno africano.

\section{AGRADECIMENTOS}

Ao Grupo Massafra por disponibilizar sua área para pesquisa.

\section{REFERÊNCIAS}

CAMPOS, B. P. F.; BINOTI, D. H. B. SILVA, M. P.; LEITE, H. G.; BINOTI, M. L. M. S. Efeito do modelo de afilamento utilizado sobre a conversão de fustes de árvores em multiprodutos. Scientia Forestalis, Piracicaba, v. 42, n. 104, p. 513-520, dez. 2014.

CAMPOS, J. C. C.; LEITE, H. G. Mensuração florestal: perguntas e respostas. 5 ed. Editora UFV, Viçosa: UFV, 2017. 605 p. 
CAMPOS, B. P. F.; SILVA, G. F.; BINOTI, D. H. B.; ADRIANO RIBEIRO DE MENDONÇA, A. R.; LEITE, H. G. Descrição do perfil do tronco de árvores em plantios de diferentes espécies por meio de redes neurais artificiais. Pesquisa florestal brasileira, Colombo, v. 37, n. 90, p. 99-107, abr./jun. 2017

CHICHORRO, J. F.; RESENDE, J. L. P.; LEITE, H. G. Equações de volume e de taper para quantificar multiprodutos da madeira em floresta Atlântica. Árvore, v.27, n.6, p.799-809, 2003.

CORDEIRO, M. A; PEREIRA, N. N. DE J.; BINOTI, D. H. B.; BINOTI, M. L. M. DA S.; LEITE, H. G. Estimativa do volume de Acacia mangium utilizando técnicas de redes neurais artificiais e máquinas vetor de suporte. Pesquisa florestal brasileira, Colombo, v. 35, n. 83, p. 255261, jul./set. 2015.

COSTA, E. A.; FINGER, C. A. G.; SCHNEIDER, P. R.; HESS, A. F. Função de afilamento e sortimentos de madeira para Araucaria angustifolia. Ciência Florestal, Santa Maria, v. 26, n. 2, p. 523-533, abr.-jun., 2016.

ESPÍRITO-SANTO, F. D. B.; SHIMABUKURO, Y. E.; ARAGÃO, L. E. O. C. de; MACHADO, E. L. M. Análise da composição florística e fitossociológica da Floresta Nacional do Tapajós com o apoio geográfico de imagens de satélites. Revista Acta Amazônica, Manaus, v. 35, n. 2, p. 155-173. 2005.

FIGUEIREDO FILHO, F.; RETSLAFF, F. A. S.; KOHLER, S. $V_{\text {.; }}$ BECKER, $M_{\text {; }}$
BRANDES, D. Efeito da Idade no Afilamento e Sortimento em Povoamentos de Araucaria angustifólia, Floresta e Ambiente, v. 22, n. 1, p. 50-59, 2015.

LEITE, H.G.; SILVA, M.L.M.; BINOTI, D.H.B.; FARDIN, L.; TAKIZAWA, F.H. Estimation of inside-bark diameter and heartwood diameter for Tectona grandis Linn. trees using artificial neural networks. European Journal of Forest Research, v. 130, n. 2, p. 263-269. 2011.

PINHEIRO, A. L.; COUTO, L.; PINHEIRO, D. T.; BRUNETTA, J. M. F. C. Ecologia, silvicultura e tecnologia de utilização dos Mognos-Africanos (Khaya spp.). $1^{a}$ ed. Sociedade Brasileira de Agrossilvicultura - Viçosa, MG, 2011. $102 \mathrm{p}$.

RIBEIRO, A.; FERRAZ FILHO, A. C.; SCOLFORO, J. R. S. O Cultivo do Mogno Africano (Khaya spp.) e o Crescimento da Atividade no Brasil. Floresta e Ambiente, v. 24, e00076814, p.1-11. 2017.

SCHRÖDER, T.; HOFIÇO, N. A. S.; ZIMMERMANN, A. P. L.; PEREIRA, L. D.; ROCHA JUNIOR, D. S.; MEYER, E. A.; FLEIG, F. D. Métodos de estimativa de volume comercial para Eucalyptus grandis: especificidades e recomendações. Pesquisa Florestal Brasileira, Colombo, v. 33, n. 73, p. 1-7, 2013. 